Fecha de recepción: septiembre 2019 Fecha de aceptación: noviembre 2019 Versión final: febrero 2020

\section{El Taller Integrado de Diseño como espacio de aprendizaje y simulador de vuelo}

Osvaldo Zorzano ${ }^{(1)}$

Resumen: ¿Cómo y en qué momento dar sentido de la realidad a un estudiante de Diseño? ¿En qué espacios se pueden poner en práctica en contextos reales los conocimientos y competencias adquiridas por un estudiante? ¿Qué procesos y herramientas pedagógicas utilizar para que el estudiante ejerza la autonomía que necesitará para enfrentar un Proyecto de Título?

Estas preguntas rondan en la cabeza de muchos académicos y docentes y son parte de las discusiones que se dan dentro de las unidades académicas cuando se discute el sentido y propósito de formar diseñadores (o deberían serlo). Un Taller de Diseño es siempre un espacio de aprendizaje donde convergen tradiciones y modelos históricos de "cómo debe transmitirse el conocimiento a la hora de formar colegas" versus "cómo innovar permanentemente e integrar las nuevas herramientas y lenguajes" que permiten estar a tono con el contexto en que estos jóvenes colegas ejercerán la profesión. Este desafío se vuelve más rudo cuando se trata del taller que "puentea" el cierre del pregrado con la etapa de titulación.

El presente artículo es un registro muy resumido de la experiencia de Taller Integrado de Diseño en el octavo semestre de la carrera de Diseño en la Universidad del Desarrollo.

Palabras clave: Taller de Diseño - transdisciplinariedad - aprendizaje significativo - aprender haciendo - metodología Compass.

[Resúmenes en inglés y portugués en las páginas 241-242]

(1) Osvaldo Zorzano es diseñador y docente dela Universidad del Desarrollo desde el año 2002. Pertenece a las áreas tecnológica y proyectual de la carrera. Ha impartido diversos talleres desde 2006 y forma parte del equipo de Taller Integrado desde 2014. Su visión de la enseñanza del Diseño siempre se ha centrado en el uso experimental y la vez crítico de las tecnologías y en rol del diseñador como generador consciente y responsable de cultura material. 


\section{Introducción}

El octavo semestre de la malla de estudios de pregrado de Diseño en la Universidad del Desarrollo (UDD) contempla un último Taller de Diseño, llamado Taller Integrado de Diseño (TID) que funciona de manera convergente para las tres menciones impartidas por la Facultad: Diseño de Ambientes y Objetos, Diseño Digital y Diseño Gráfico. Tanto los (y las) estudiantes como los (y las) docentes a cargo provienen de todas las menciones y se combinan de manera estratégica, buscando siempre tres virtudes: amplitud y convergencia de miradas; complementación de saberes disciplinares; y el valor de la experiencia de cada integrante como un plus en sí mismo. Los equipos de cada sección de este taller (generalmente son entre cinco y seis secciones) están compuestos por una dupla de profesores, sin ayudante y alrededor de 20 alumnos distribuidos equitativamente entre las tres menciones, siendo la con menos representantes la de Digital (actualmente Diseño de Interacción), históricamente.

Se trabaja en torno a un tema troncal o paraguas (o más de uno), abordado simultáneamente por todas las secciones, tomando cada una la dirección que el equipo (docentes y estudiantes) decide darle. Este tema se mantiene durante todo un semestre; es decir, proyectos de largo aliento. Eso implica un desafío en cuanto a la sincronía versus la autonomía de cada sección. Las temáticas abordadas año tras año varían según los intereses de la Facultad, la contingencia, las oportunidades que se presentan y la mantención del sentido de este taller.

Se ha trabajado con museos, instituciones sin fines de lucro, fundaciones, municipalidades, etc. Este componente es uno de los ejes de este espacio de aprendizaje, pues el "sentido de realidad" que se busca dar a la experiencia de pasar por este taller de último semestre es muy valioso, tanto para los estudiantes como para el equipo docente. Los resultados a los que se llega son siempre abiertos (y, generalmente, definidos por los propios estudiantes) y quedan disponibles para ser aprovechados por nuestras contrapartes año a año.

Si bien un taller no es un espacio vinculado necesariamente al uso de tecnologías, en este caso los estudiantes son alentados a incorporarlo al proceso de diseño como una herramienta de visualización y prototipado. No se enseñan programas ni uso de hardware dentro del taller, pero sí se entrega retroalimentación sobre cómo optimizar su empleo, se reflexiona acerca de su utilización y sentido, y se ayuda a solucionar problemas derivados de la incorporación de dispositivos como cortadoras láser e impresoras 3D, entre otros. El relato que prosigue es el testimonio de la experiencia de cinco años impartiendo esta asignatura; abriendo y guiando este espacio de aprendizaje y convivencia. Por esto, el registro y el testimonio se combinan de manera fluida en las líneas que siguen.

\section{El TID dentro del itinerario de Pregrado}

Tal como ya se ha mencionado, el "Integrado" (como suelen llamarle los estudiantes en coloquial), es una asignatura de la Línea Proyectual en las tres menciones de la carrera de Diseño, ubicada en el octavo semestre. Es la asignatura de mayor creditaje y una de 
las que más horas directas implica (dos sesiones de 4,5 horas cronológicas a la semana, durante 16 semanas). Al aprobar este taller, más todos los cursos del nivel, los estudiantes quedan habilitados para iniciar la etapa final de Proyecto de Título, conducente al grado de licenciado y al título profesional de Diseñador(a) en la mención que corresponda. Las instancias de calificaciones varían de sección en sección, pero por lo general se componen de una serie de entregas parciales (correspondientes al 70\% de la ponderación total) y un examen final (correspondientes al 30\% restante).

Desde el punto de vista formativo, este espacio de aprendizaje es la transición entre la etapa formativa más centrada en entregar contenidos y encargos y la etapa de autonomía total que implica el Proyecto de Título. Este proceso se da mediante la autodefinición de encargos, objetivos y entregables por parte de los equipos de estudiantes, quienes se organizan en una metáfora de lo que sería una "miniagencia" de Diseño, tratando de combinar integrantes de todas las menciones (cosa que no siempre se logra por la asimetría numérica de ellas). Estos equipos enfrentan un desafío paraguas o troncal que viene dado desde la Facultad de Diseño. El del año pasado, por ejemplo, fueron los 17 Objetivos de Desarrollo Sostenible de la ONU. Desde ese pie forzado, los equipos deben detectar el marco de proyecto en torno al cual desarrollarán sus propuestas durante el semestre. Este marco es un triángulo (a veces de Las Bermudas) entre: oportunidad/problema; entorno de intervención; red de actores clave. Según este contexto autodefinido (y aprobado por el equipo docente), se generan los objetivos de proyecto, se definen los entregables y se fijan las etapas de desarrollo con sus respectivos hitos a evaluar.

Para que tanta autonomía y diversidad dentro del Taller tengan cierta estabilidad y estructura, se necesita una columna vertebral. Para el TID, esta columna es la metodología de Diseño. Esta debe ser clara, compartida y apropiable por los equipos. Desde el año pasado, dentro de nuestra sección hemos adoptado una versión custom de la metodología Compass de la fundación INDEX: Design to Improve Life. Cada etapa de esta metodología se cierra con una entrega y una calificación parcial. Una vez entregada la nota por parte de los docentes (que en nuestra escala va de 1,0 a 7,0, siendo 4,0 el nivel de aprobación mínimo), los equipos tienen la opción de diferenciar sus notas individuales, manteniendo como promedio la nota entregada. Finalmente, todas las secciones pasan por un examen de cierre del taller, que se realiza en un mismo día y de manera consecutiva, frente a una comisión de docentes externa al equipo de Taller Integrado. A esta instancia son invitadas las instituciones que han sido contraparte durante el semestre y se evalúa de manera transversal a todos los equipos en una jornada que puede extenderse por medio día. Quienes aprueban y tienen todas sus asignaturas al día, se convierten en egresados de la carrera de Diseño.

\section{El equipo que conforma el Taller y su estructura}

Tal como se mencionó anteriormente, el equipo docente que compone este Taller está conformado por diseñadores de diversas especialidades y perfiles, pero también por arquitectos y sociólogos. Este equipo se subdivide en duplas, tratando de combinar disciplinas, miradas y perfiles profesionales y personales. En mi caso, mis partners han sido un soció- 
logo (profesor invitado extranjero) y un arquitecto, cuyas competencias y experiencias resultan muy complementarias a las mías, más orientadas al área de tecnologías y producción. Cada dupla tiene la oportunidad de darse a conocer a la generación de estudiantes antes de empezar los talleres, a fin de que cada cual pueda optar a los talleres que más le atraen o identifican.

El equipo se comunica por canales formales e informales. Existen correos y documentos de coordinación que provienen desde la Facultad de Diseño, pero también están las aplicaciones como Whatsapp que nos permiten mantener el contacto "en caliente" durante el semestre. Para el equipo docente es importante poder mantener cierta sincronía, dentro de la libertad de cátedra de cada sección, y poder prever y organizar las fechas clave durante el semestre.

Dentro de cada sección, los estudiantes se organizan en equipos casi desde el principio. Pueden ser duplas, tríos o cuartetos, pero siempre alentándolos a mezclar menciones y pidiéndoles que asuman roles y responsabilidades individuales dentro del equipo, lo que permite una organización y evaluación diferenciada más clara, tanto para ellos como para nosotros.

Existe un tercer actor clave dentro de esta organización, que se hace presente solamente dos o tres veces durante el semestre, pero cuya participación es gravitante. Se trata de una comisión evaluadora externa al equipo docente del TID y que, por lo general, está compuesta por las autoridades de la Facultad. Esta comisión se constituye para evaluar a los equipos cuando estos presentan sus propuestas a nivel incipiente y, luego, al final, en el examen de la asignatura para medir el avance y calidad de las propuestas finales.

\section{Descripción del taller como espacio de aprendizaje formal}

Durante las 16 semanas que dura esta asignatura, los equipos desarrollan sus propuestas, literalmente desde cero. En la reunión de inicio del TID se presenta el equipo docente y la temática paraguas del semestre. Desde que se conforman las secciones y el taller empieza a trabajar, se abren dos espacios de aprendizaje formal: uno es el del conocimiento instruido; y el otro, del construido. El primero dice relación con contenidos enseñados en clase como, por ejemplo: cómo analizar y clasificar referentes, cómo hacer un benchmark, cómo construir un mapa de actores clave, cómo elaborar presentaciones persuasivas, entre otros. El segundo espacio de aprendizaje es tal vez el más significativo, pues se construye desde la experiencia personal y es el que tiene que ver con "salir a la calle" y encontrar asesores, contrapartes, potenciales proveedores para la serialización de un producto y usuarios para testeos y evaluación de prototipos. Esta parte del taller es fundamental pues le da un sentido de realidad que ningún apunte o clase magistral les puede transferir. Es la oportunidad de comprender cómo un diseñador opera como un mediador entre una institución y las personas que se benefician de ella; entre una solución a nivel de prototipado y la serie que se generará si es aprobada. Son esos desafíos los que enfrentarán tras la universidad, evento que ocurrirá un año después de aprobar este taller. Pero no solo eso; mucha de la metodología que ahora se les inculca como equipo dentro del TID la utilizarán de manera 
autónoma al año siguiente, en su proceso de titulación. Por estos motivos, el incentivo a la autonomía y el sentido de realidad son tan enfáticos en este curso.

La evaluación dentro del taller, por todo lo expuesto anteriormente, es un tema delicado. $\mathrm{Al}$ no haber contenidos de tipo "materia que aprender", no es fácil medir el grado de aprendizaje con las fórmulas tradicionales. Sin embargo, existen dos criterios estables que los estudiantes comprenden bien, como un horizonte de resultados esperados. El primero es el grado de integración de los contenidos instruidos al proyecto que cada equipo está desarrollando. El segundo es el cumplimiento con los objetivos y los entregables que cada equipo compromete (coherencia y suficiencia). Finalmente, un tercer aspecto se agrega a esta rúbrica sostenida; la calidad "profesional" de todo lo entregado o mostrado: presentaciones, prototipos y maquetas, piezas gráficas, etc. Para ser consistentes en el eje de la autonomía, parte de esta evaluación es de autoevaluación la que se da de dos formas. La primera es que los mismos estudiantes se evalúen, contrastando lo entregado con lo comprometido y convirtiendo eso en una calificación donde se busca el balance ente el amor propio (y el apego a las buenas calificaciones) y el sentido de autocrítica. La segunda es "entregar la nota (o calificación)" al equipo y que sus integrantes tengan derecho a notas diferenciadas cuyo promedio es la nota puesta por los profesores. Esto permite al equipo ajustar cuentas internas en beneficio de algún miembro o perjuicio de otro. Demás está decir que la posibilidad de dejar todas las notas parejas está disponible y es lo que suele suceder.

\section{El taller como simulador de vuelo}

Esta búsqueda de la autonomía y sentido de la realidad requiere de un espacio de aprendizaje extramuros donde la formalidad y estructura no necesariamente son las de la academia. No sirve tener buenas calificaciones para ser creíble ante un profesional que no pertenece al mundo de la pedagogía y que se mueve en el entorno laboral. Pero ser creíble para un potencial cliente, socio estratégico o proveedor es fundamental para un diseñador, sobre todo si piensa ser freelance. Es por esto que el taller potencia permanentemente la política "vaya allá afuera y pregúntele al que sabe". Esto ha llevado a nuestros estudiantes a contactar aliados en municipalidades, ONG, empresas privadas de diferentes tamaños, profesionales destacados en diferentes áreas e incluso entidades gubernamentales como ministerios. El impacto positivo que tiene el éxito en establecer estas relaciones profesionales es un potenciador y un validador real que supera en muchos sentidos a una buena nota. Pero, sobre todo, permite que los estudiantes empiecen a desarrollar las competencias que la universidad como entorno protegido no les puede entregar: credibilidad, validación, capacidad de negociación, entre otras.

Por eso junto a mi dupla, el profesor y arquitecto Mauricio Acuña, le llamamos a este espacio de aprendizaje "el simulador de vuelo", donde te puedes estrellar y volver a empezar. Donde los errores, si bien no salen gratis (porque todo tiene consecuencias y eso también se aprende), no tienen las secuelas que tendrían en la vida real. 


\section{Horizontes de resultados esperados}

Un taller que es así de complejo implica muchos aprendizajes, muchos resultados y muchos ámbitos a evaluar. Por eso mismo, es más fácil comprender esta multidimensionalidad de resultados esperados si se separan por ámbito:

\section{Sobre los entregables}

Considerando que se trata del último taller del pregrado (y la antesala al proceso de titulación), todo es "cobrable", desde los errores de ortografía en las presentaciones (que llegan a niveles preocupantes a veces) hasta las finas terminaciones del oficio en maquetas y prototipos, pasando por la puntualidad, la coherencia entre lo que se dice y lo que se hace; lo que se ofrece y lo que se entrega. En ese sentido, el TID es de alta exigencia (y, en ocasiones, estrés). Las excusas no equivalen a los resultados. El nivel no es el más alto de un estudiante... Es el mínimo que se espera de un profesional, porque "estudiante" no es su profesión. Son diseñadores.

Dentro de este ámbito, una mención especial merece el uso de tecnologías. Si bien no es un taller centrado en ellas, su uso e incentivo es permanente, pues los diseñadores de hoy deben integrarlas a su ejercicio profesional de manera instrumental y natural. Conocer las tecnologías de producción y prototipado, las técnicas de maquetaje de alto realismo y mock-ups convincentes es fundamental a la hora de presentar un proyecto. En el Taller no se enseñan programas, pero sí se discute sobre su autoaprendizaje, uso óptimo de herramientas digitales y cómo esto modifica el perfil y las competencias del diseñador que los usa con un discurso claro.

\section{Sobre la metodología de Diseño}

Parte del desarrollo del proyecto troncal del Taller es instruido y guiado por el equipo docente; la otra mitad es decidida y manejada por cada equipo de diseñadores (estudiantes) lo más autónomamente posible. Ambas instancias se fusionan de manera permanente durante el curso. El hilo conductor que permite que esto ocurra es la metodología de diseño que, como se dijo anteriormente, se basa en Compass, pero se adapta según cómo se va dando la asignatura. Esta metodología que va desde el levantamiento de antecedentes -para comprender dónde está el problema u oportunidad de Diseño- hasta el registro y comunicación testeo y validación (junto a una evaluación de resultados), es un proceso que se hace de manera muy reflexiva y tratando de que los estudiantes comprendan por qué cada paso es necesario, pero no puede aplicarse como una receta, sino que se trata de un proceso. Los estudiantes que se apropien de este proceso podrán (y deberán) aplicarlo el semestre siguiente, durante su proyecto de título y podrán verificar en un corto plazo cuán útil es guiarse por una metodología probada anteriormente. 
Para poder evaluar este aspecto del curso es necesario que los estudiantes expresen de qué manera han empleado la metodología. Es una especie de rendición de cuentas, pero usando lenguaje de diseñador (bocetos, renders, gráficos, infogramas, etc.)

\section{Sobre la comunicación de los proyectos}

El taller se cierra con una presentación de pocos minutos que resume el semestre, el desarrollo del proyecto y los resultados preliminares obtenidos de las experiencias de testeo y validación $(\mathrm{T}+\mathrm{V})$ de la propuesta. Esta presentación es el registro no solo del proyecto, sino de la experiencia personal que implica para cada miembro de los equipos. Es testimonial y documental al mismo tiempo. En ella, la coherencia, la eficiencia y la credibilidad en la rendición de cuentas son fundamentales. Todos son principios del ejercicio de la profesión con sentido de ética y excelencia.

En esta evaluación, que es la última antes del examen, se consideran principalmente tres aspectos. El primero es "Uso de lenguajes y códigos de comunicación" (visual o audiovisual). El segundo, "Registro completo y claro del proceso". Y tercero: "Oficio en todo lo presentado, incluyendo su propia exposición oral y presencia".

\section{Sobre la autonomía y el sentido de realidad}

Tal vez el aprendizaje más trascendente del TID es comprendido después de haberlo aprobado. Se trata de la genuina sensación de que el diseñador está listo para cerrar su pregrado. Ha adquirido la capacidad de gestionar sus propios proyectos y conformar su red de apoyo y de actores clave; de comprender cómo llegar a una propuesta, cómo presentarla y posteriormente cómo producirla o ejecutarla. Claramente este aprendizaje no se puede medir de manera total durante el curso. Solo se puede evaluar el proceso por el que cada estudiante va pasando y sus propias reflexiones. Este ámbito requiere de mucho análisis, conversación y guía por parte del equipo docente para que lo aprendido se vuelva autoevidente para los estudiantes. Entender para qué se diseña, con qué recursos se dispone y cómo plantear y defender una propuesta son reflexiones necesarias para que el joven diseñador "realice un boceto con un sentido de realidad" desde el inicio. Esta evaluación recae fuertemente en los propios estudiantes. Se trata de una autoevaluación que apela a la madurez, sinceridad, autocrítica y autoestima de cada persona. Una cosa que se les recalca a los jóvenes diseñadores es que "no se aprende para aprobar; se aprende para ejercer".

\section{La experiencia desde el lado del aprendiz}

Es muy importante realizar periódicas evaluaciones del taller como espacio de aprendizaje, pero también de convivencia. Plantear los temas a evaluar de manera abierta y franca, en desayunos o conversaciones en el pasto (tal cual). Y ser muy humildes y poco defensivos a la hora de escuchar. Aflorarán los elogios, pero también las críticas (más necesarias que los 
elogios, para mejorar). Para los estudiantes es un espacio que no busca desquites o "cobranzas", pues el taller los ha hecho partícipes de su propio proceso formativo. Tanto los compromisos como los objetivos de cada equipo han sido autopropuestos, por lo que la vara no la ha fijado el equipo docente. Esto les permite analizar el TID con mucha sinceridad. Sostenidamente (en estos cinco años), la experiencia es valorada y evaluada como el taller más duro, pero del que más se aprende. La experiencia más estresante por la sensación de que "están solos y todo depende de ellos y su capacidad de articular su red de actores clave" (y tienen mucha razón). Valoran mucho la convergencia de menciones y los entregables que se pueden desarrollar gracias a esa combinación. También valoran mucho la libertad y la confianza; el trato de adultos y profesionales que se les da, dentro de los marcos de lo académico.

Obviamente no todo son elogios. Las críticas van desde los detalles mejorables fácilmente hasta aspectos medulares del TID. Las más frecuentes tienen relación con que esta experiencia sea única y al final. Muy tarde para combinar menciones, integrar actores ajenos al mundo académico y dar tanta autonomía de manera repentina. Consideran que estas tres cosas debieran hacerse antes y paulatinamente. En la malla actual es así. Hay talleres integrados desde antes y su complejidad es progresiva. También hay críticas sobre la coordinación de todo el TID como un solo gran taller, lo que complica la sincronía entre secciones. Y finalmente, la crítica de la poca continuidad de los proyectos iniciados en el TID una vez que se termina, lo que involucra a instituciones reales es tal vez la más atendible, porque para resolverla no basta con la voluntad del equipo docente.

\section{Conclusiones}

La experiencia de Taller Integrado ha sido valorada positivamente tanto por estudiantes como por la misma Facultad, por diversas razones. La primera y la más importante es porque pone entrega al estudiante un espacio de aprendizaje experimental y autónomo que potencia su madurez formativa. La segunda es que propicia el desarrollo de proyectos con sentido de realidad y contacto con contrapartes reales. Y la tercera es porque proporciona la oportunidad de adquirir las herramientas y competencias que se necesitarán al año siguiente para enfrentar el proceso de titulación.

Del lado de las críticas, tal vez las más recurrentes y atendibles se hallan dos. Por un lado, la que dice se refiere a la relación con lo complejo que es mantener la sintonía y sincronía de todas las secciones que componen este taller y lo desgastante que pueden llegar a ser aspectos que debieran ser secundarios como la coordinación, las comunicaciones internas y la formalidad de las entregas. Y por otro lado, tal vez la más importante, la que dice que la relación entre el esfuerzo enorme que significa sacar adelante el proyecto central para cada equipo, para que luego todo termine en una entrega a puertas cerradas y una evaluación. En este sentido, la continuidad de los proyectos o por lo menos de los temas es algo deseable. Instancias como proyectos de Título, prácticas profesionales o incluso encargos freelance podrían ser vías de continuidad que permitirían además llevar los productos desarrollados a ver la luz en el medio, fuera de la universidad. 
Abrir un espacio de esta naturaleza requiere de tres cosas. La primera es la voluntad de la unidad académica que lo administra, pues la organización y seguimiento del taller debe ser permanente y cuidadosa, ojalá a manos de una coordinación directa y permanente. La segunda es un equipo multidisciplinario que se conozca y se integre como uno solo, subdividido en duplas, a fin de que los estudiantes puedan tener revisiones y derecho a consulta no solo de los docentes en la sala, sino en todo el taller (en nuestro caso ha habido diseñadores gráficos, digitales, industriales, además de sociólogos y arquitectos). Y la tercera es un proyecto académico que dé sentido a este espacio de aprendizaje y que no sea un evento aislado, sin antesala ni secuelas. Ojalá los talleres anteriores fueran paulatinamente anticipando este taller para que cuando suceda, la adaptación no sea parte del estrés del último taller de la carrera.

\section{Referencias bibliográficas}

Albrecht, R. (1991). La evaluación formativa, un análisis crítico. Bruselas: Universidad De Boeck.

Barbier, J. M. (1993). La evaluación en los procesos de formación. Temas de Educación Paidós. Bloom, A. \& alias (1956). Taxonomy of educational objectives. NY: Longman.

Cowdroy, R. (1994). Reflections on problem based learning. Australian P.B.L. Network.

De Bono, E. (1977). Lateral Thinking. A textbook of creativity. Penguin Books, .

Lemieux, A. (1998). Théories contemporaines de l'education (4édition) Montreal: Editions Nouvelles.

Mabardi, J. F. (1997). Enjeux et perspectives de l'enseignement de l'architecture en Europe. AEEA.

Mabardi, J. F. (1997). L'enseignement du projet, Propos recueillis. Paris: L'Exote.

Maturana, H. y Varela, F. (1989). El árbol del conocimiento (5aedición) Editorial Universitaria. Von Bertalanfy, L. (1968). General system theory. NY: Braziller.

\footnotetext{
Abstract: How to give a sense of reality to design students and what time is conducive for that ? In what spaces can knowledge and skills acquired by a student be put into practice in real contexts? What pedagogical processes and tools shall we use for the student to exercise the autonomy they will need to face a Graduation Project?

These questions are around the head of many professors and teachers and they are (or should be) part of the discussions that occur within academic units when discussing the meaning and purpose of forming designers. A Design Workshop is always a learning space where traditions and historical models of "how knowledge should be transmitted when forming colleagues" versus "how to permanently innovate and integrate new tools and languages" converge to be in tune with the context in which these young colleagues will
} 
practice the profession. This challenge becomes tougher when it comes to the workshop that "bridges" the closing of the undergraduate with the degree stage.

This article is a very summary record of the experience of Integrated Design Workshop in the eighth semester of the Design degree at Universidad del Desarrollo.

Keywords: Design Workshop - Transdisciplinarity - Meaningful learning - learning by doing - Compass methodology.

Resumo: Como e a que horas dar a um estudante de design uma sensação de realidade? Em que espaços o conhecimento e as habilidades adquiridas por um aluno podem ser colocados em prática em contextos reais? Quais processos e ferramentas pedagógicas usar para o aluno exercer a autonomia necessária para enfrentar um Projeto de Título?

Essas perguntas estão na cabeça de muitos acadêmicos e professores e fazem parte das discussões que ocorrem dentro das unidades acadêmicas ao discutir o significado e o objetivo de formar designers (ou deveriam ser). Um Workshop de Design é sempre um espaço de aprendizado no qual tradições e modelos históricos de "como o conhecimento deve ser transmitido ao formar colegas" versus "como inovar e integrar constantemente novas ferramentas e linguagens" convergem para estar em sintonia com o contexto em que esses jovens colegas exercerão a profissão. Esse desafio se torna mais difícil quando se trata da oficina que "une" o fechamento da graduação com a etapa de graduação.

Este artigo é um registro muito resumido da experiência do Workshop de Design Integrado no oitavo semestre do curso de Design da Universidad del Desarrollo.

Palavras chave: Oficina de Design - transdisciplinaridade - aprendizagem significativa aprender fazendo - metodologia Compass.

[Las traducciones de los abstracts fueron supervisadas por el autor de cada artículo] 